Supporting Information

\title{
Porous Graphitic Carbons Containing Nitrogen by Structuration of Chitosan with Pluronic
}

Lu Peng, Yong Peng, Ana Primo* and Hermenegildo García*

Instituto Universitario de Tecnología Química, Universitat Politècnica de València-

Consejo Superior de Investigaciones Científicas, Av. de los Naranjos s/n, 46022

Valencia, Spain. E-mail addresses: aprimoar@itq.upv.es and hgarcia@qimn.upv.es 


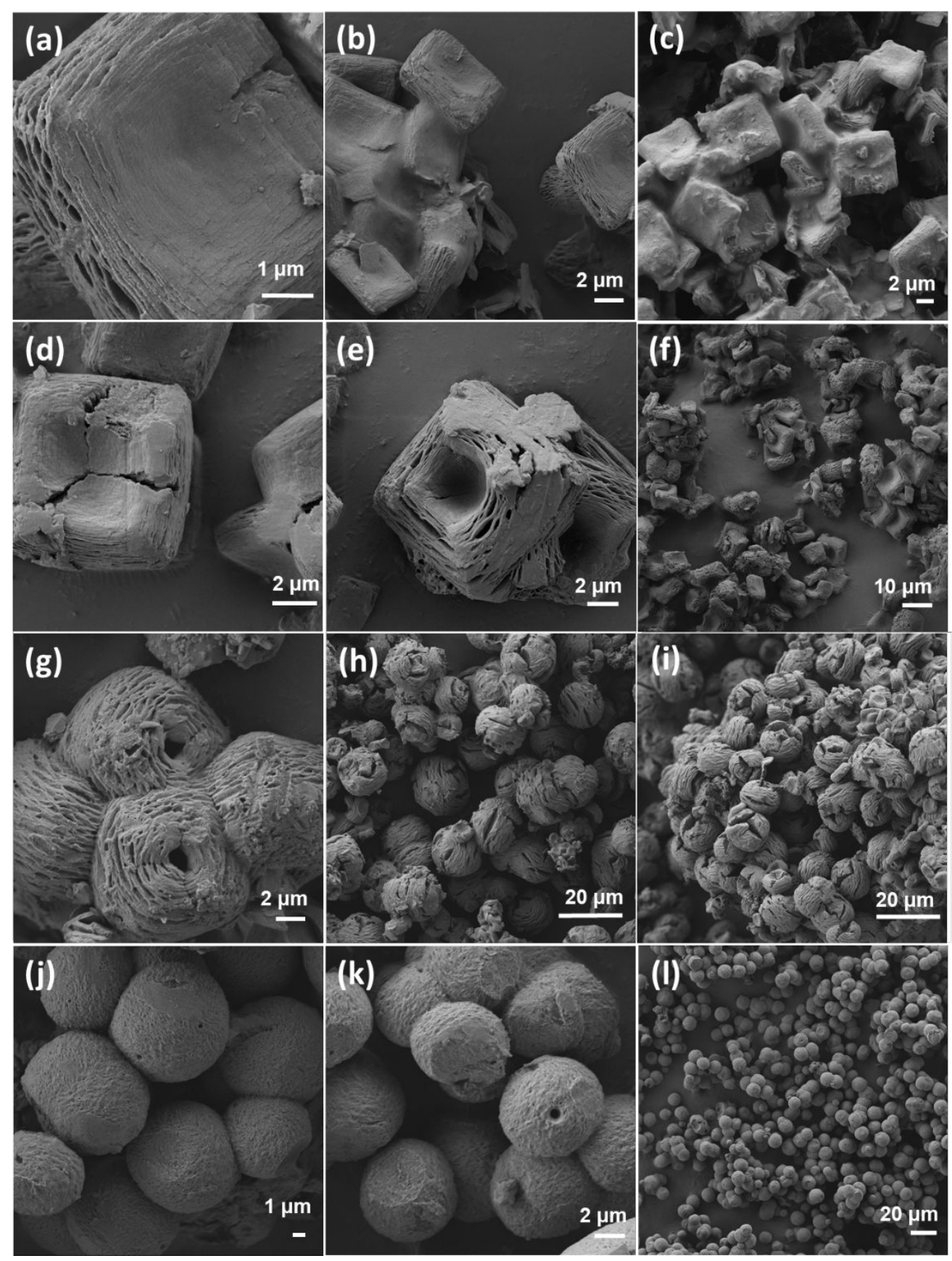

Figure S.1. . FESEM images of a, b, c) P@CS-1; d, e, f) P@CS-2; g, h, i) P@CS-3 and j, k, I) P@CS4. 


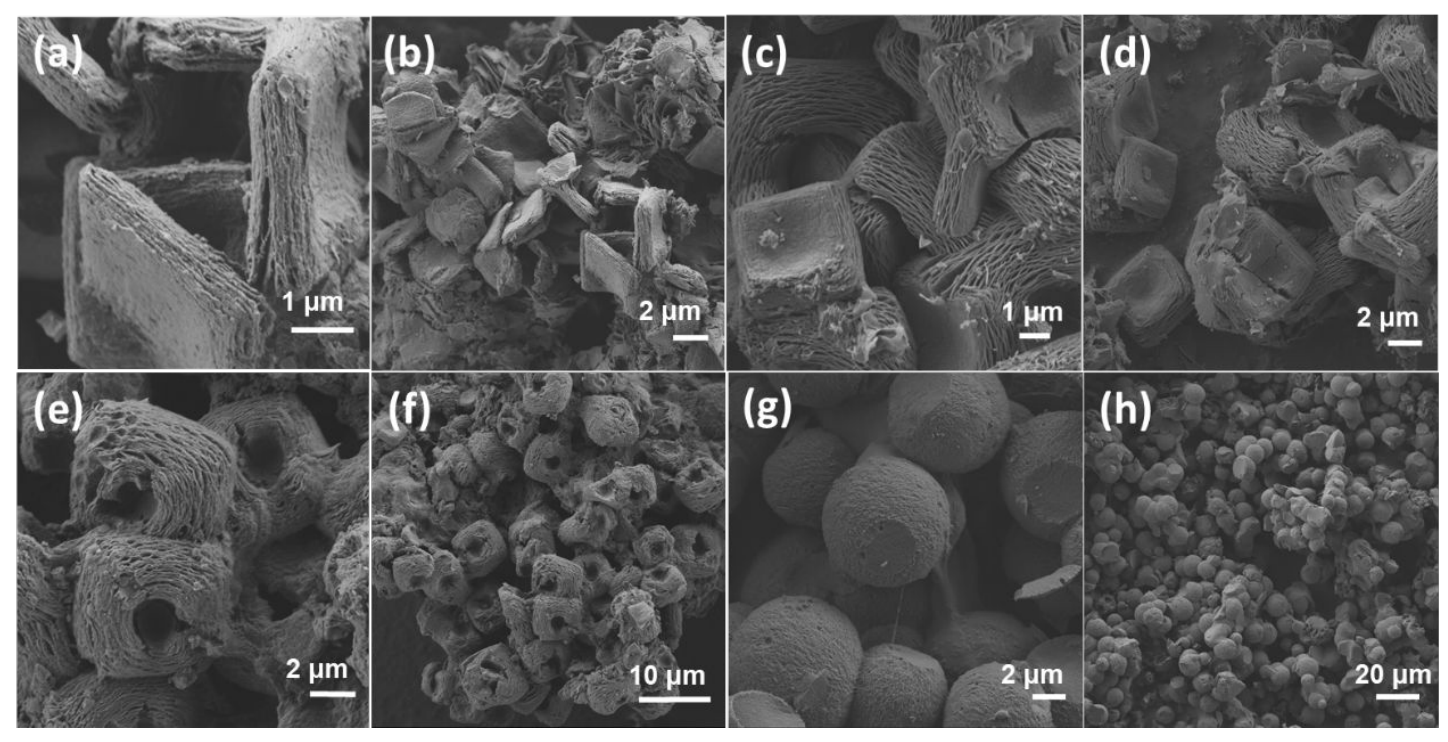

Figure S.2. FESEM images of $a, b) 3 D(N) C-1 ; c, d) 3 D(N) C-2$ e, f) 3D (N)C-3 and g, h) 3D (N)C-4. 

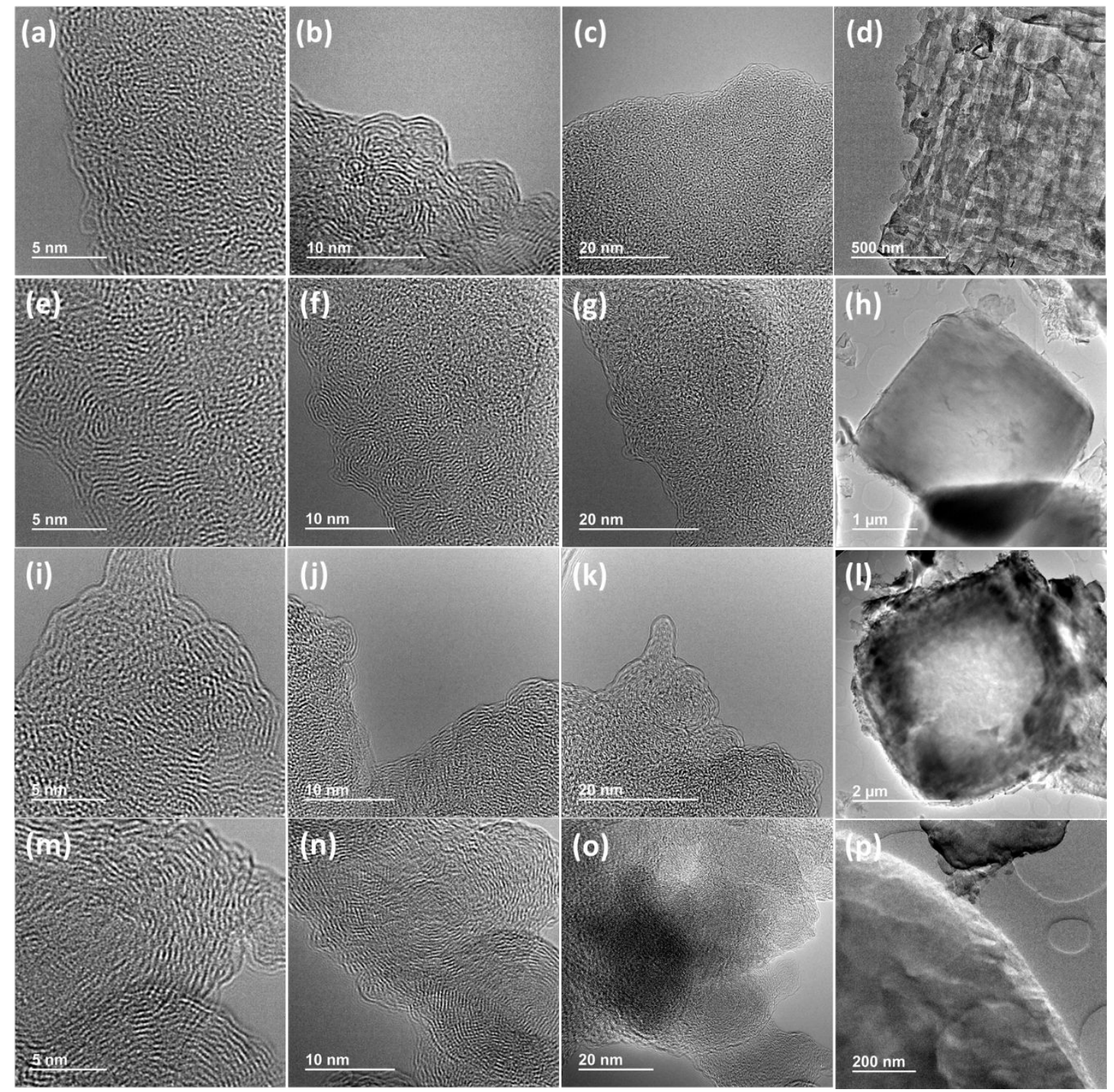

Figure S.3. TEM images of $a, b, c, d) 3 D(N) C-1 ; e, f, g, h) 3 D(N) C-2 ; i, j, k, I) 3 D(N) C-3$ and $m, n$, 0, p) 3D (N)C-4. 


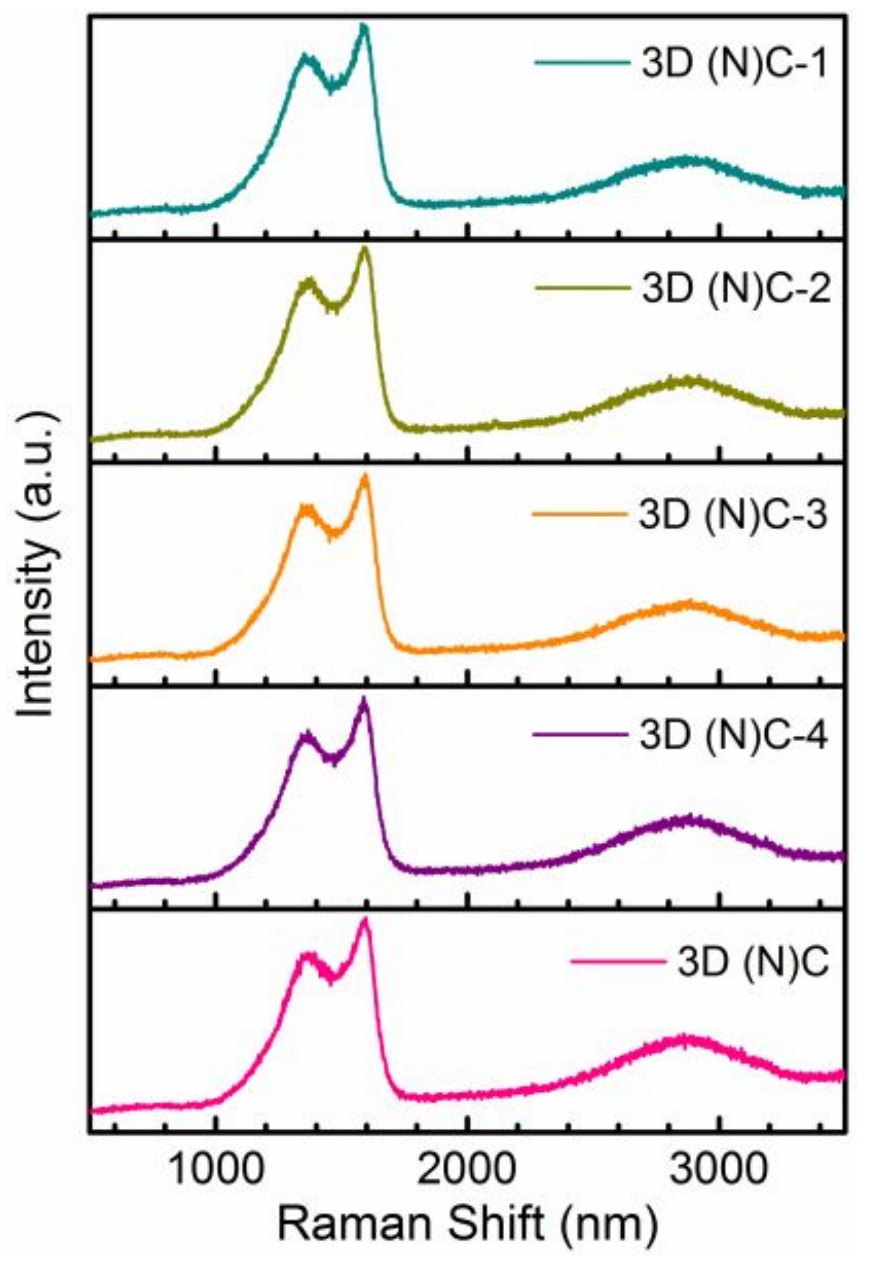

Figure S.4. Raman spectrum of 3D (N)C-n. 3D (N)C was prepared without Pluronic P123. 
(a)

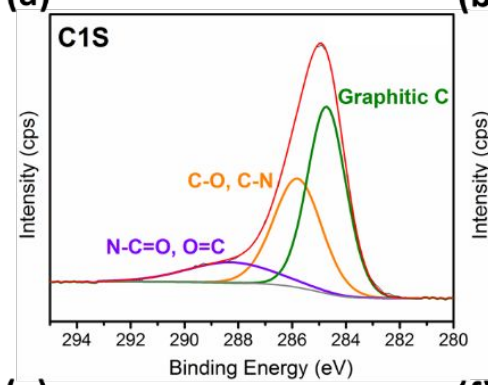

(e)

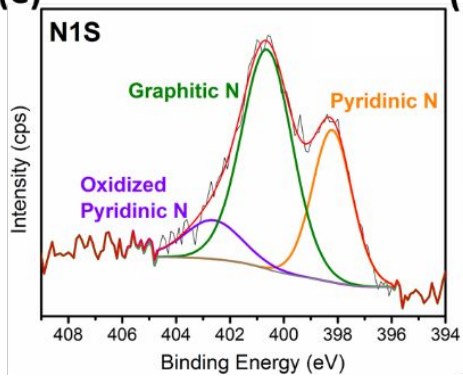

(b)

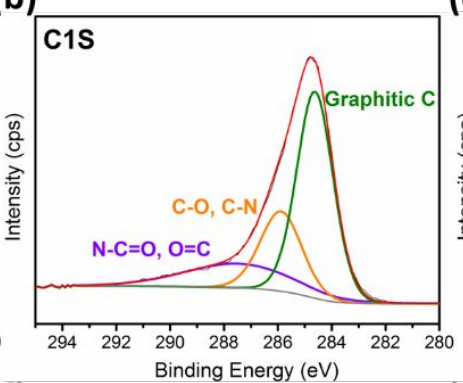

(f)

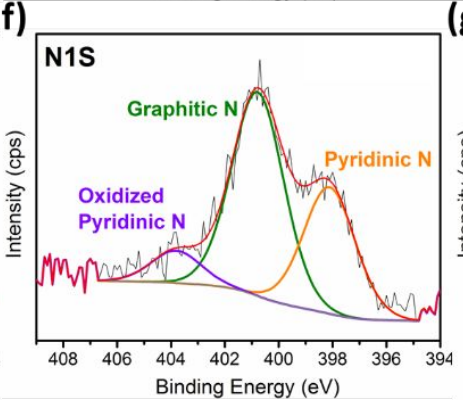

(c)

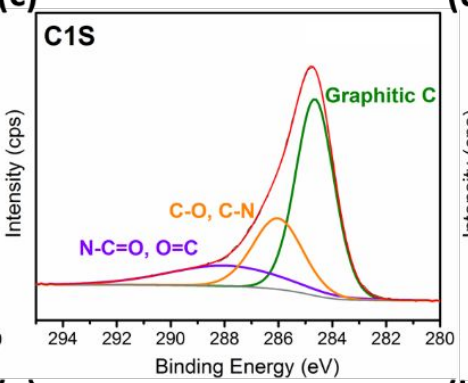

(g)

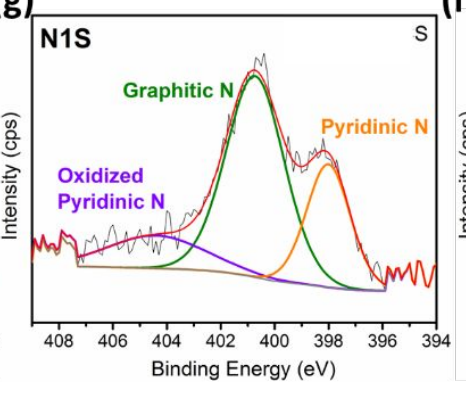

(d)

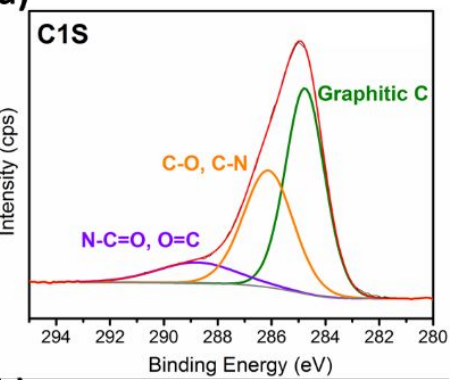

(h)

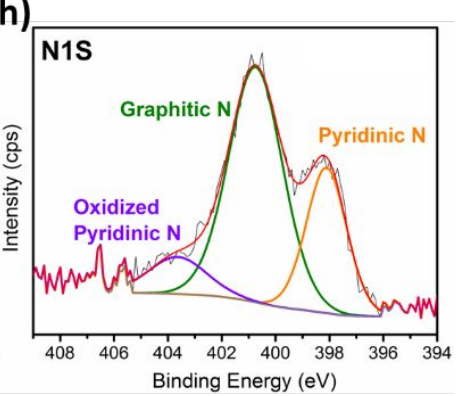

Figure S.5. High-resolution XPS C1s spectrum and N1s spectrum of a), e) 3D (N)C-1; b, f) 3D (N)C-

3; C, g) 3D (N)C-4 and d, h) 3D (N)C.
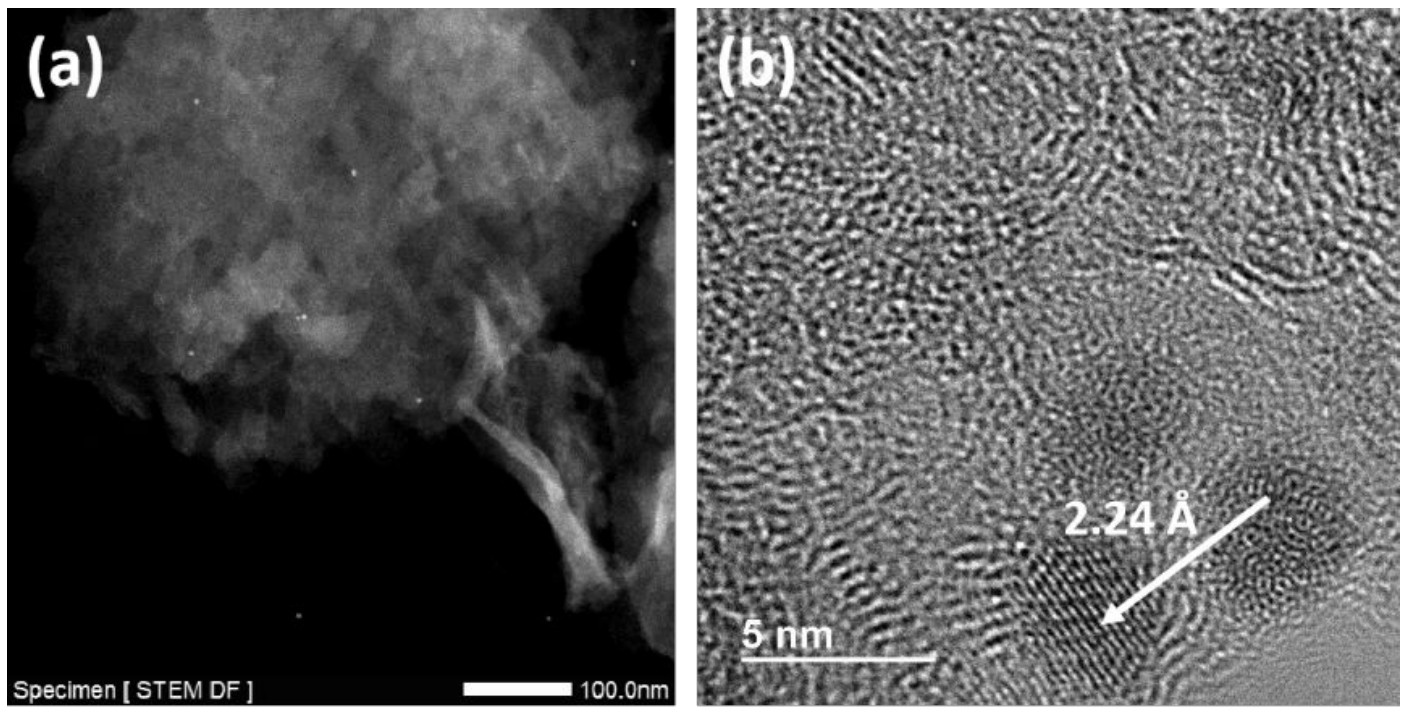

Figure S.6. a) High-angle annular dark-field (HAADF) images taken in STEM mode and b) HRTEM images taken in transmission mode of $3 \mathrm{D}(\mathrm{N}) \mathrm{C}-2$ after irradiation in the presence of $\mathrm{H}_{2} \mathrm{PtCl}_{6}$, where the formation of small Pt nanoparticles can be clearly observed after irradiation. 

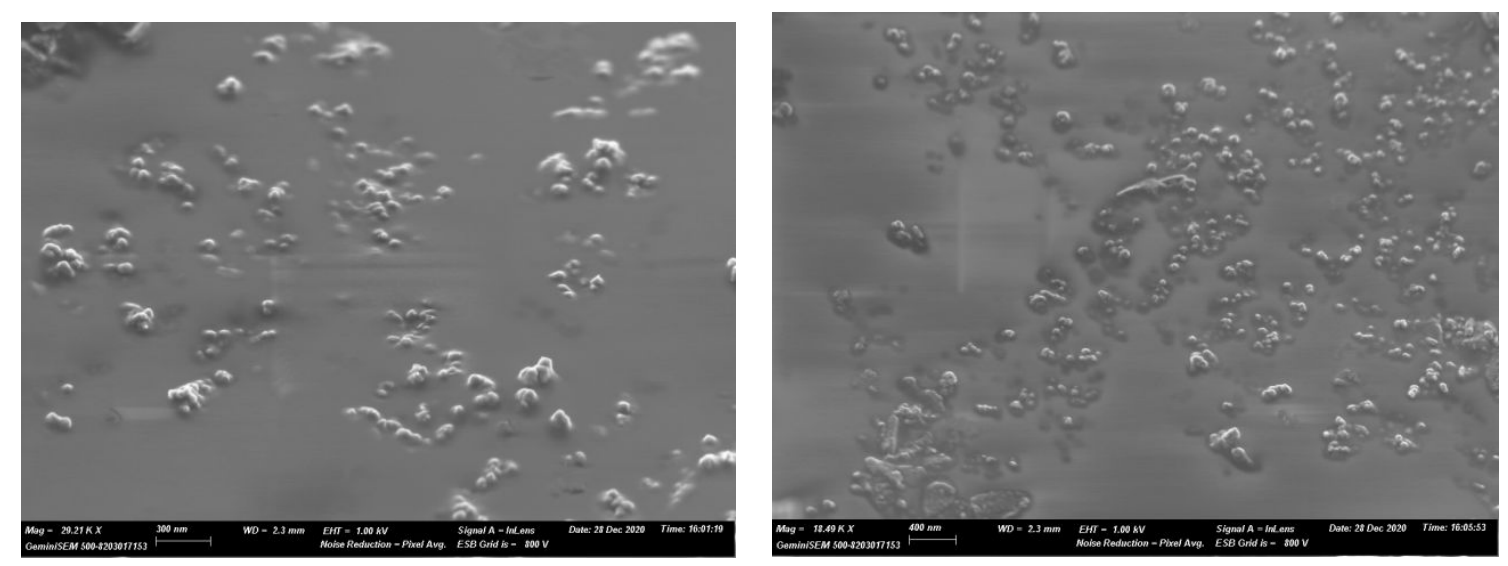

B
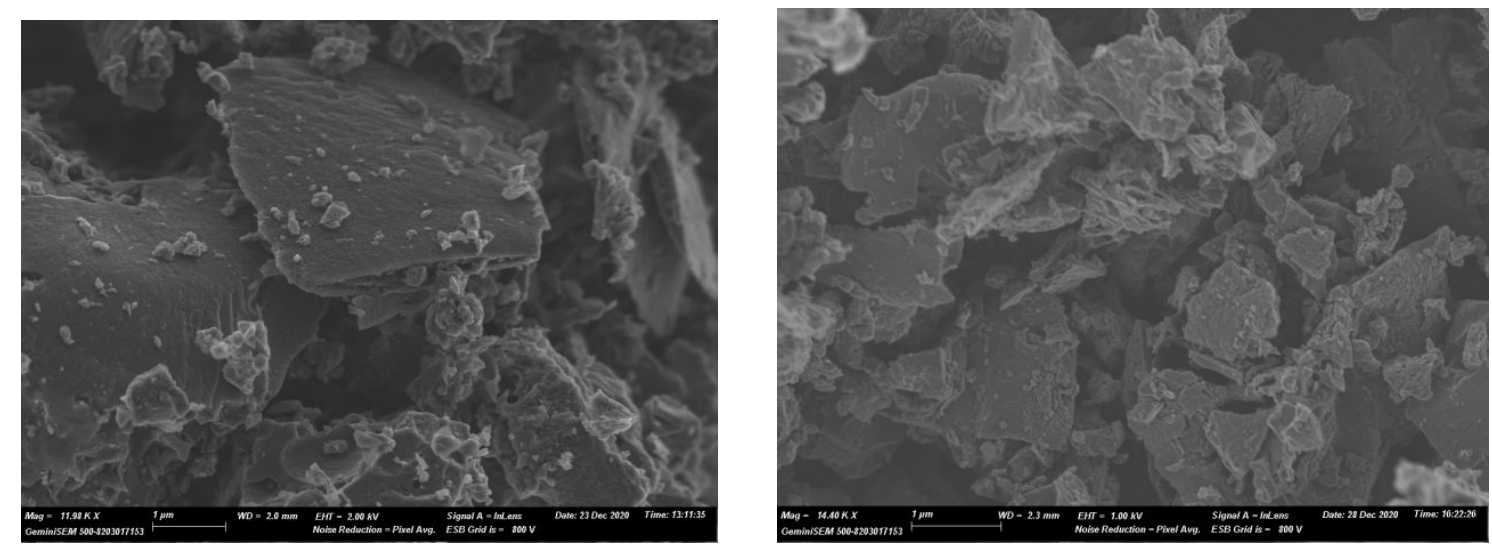

Figure S7. FESEM images of the material present in the supernatant (A) and the sediment (B) after sonication of 3D (N)C-2 and allowing the dispersion to sediment for $5 \mathrm{~h}$. 


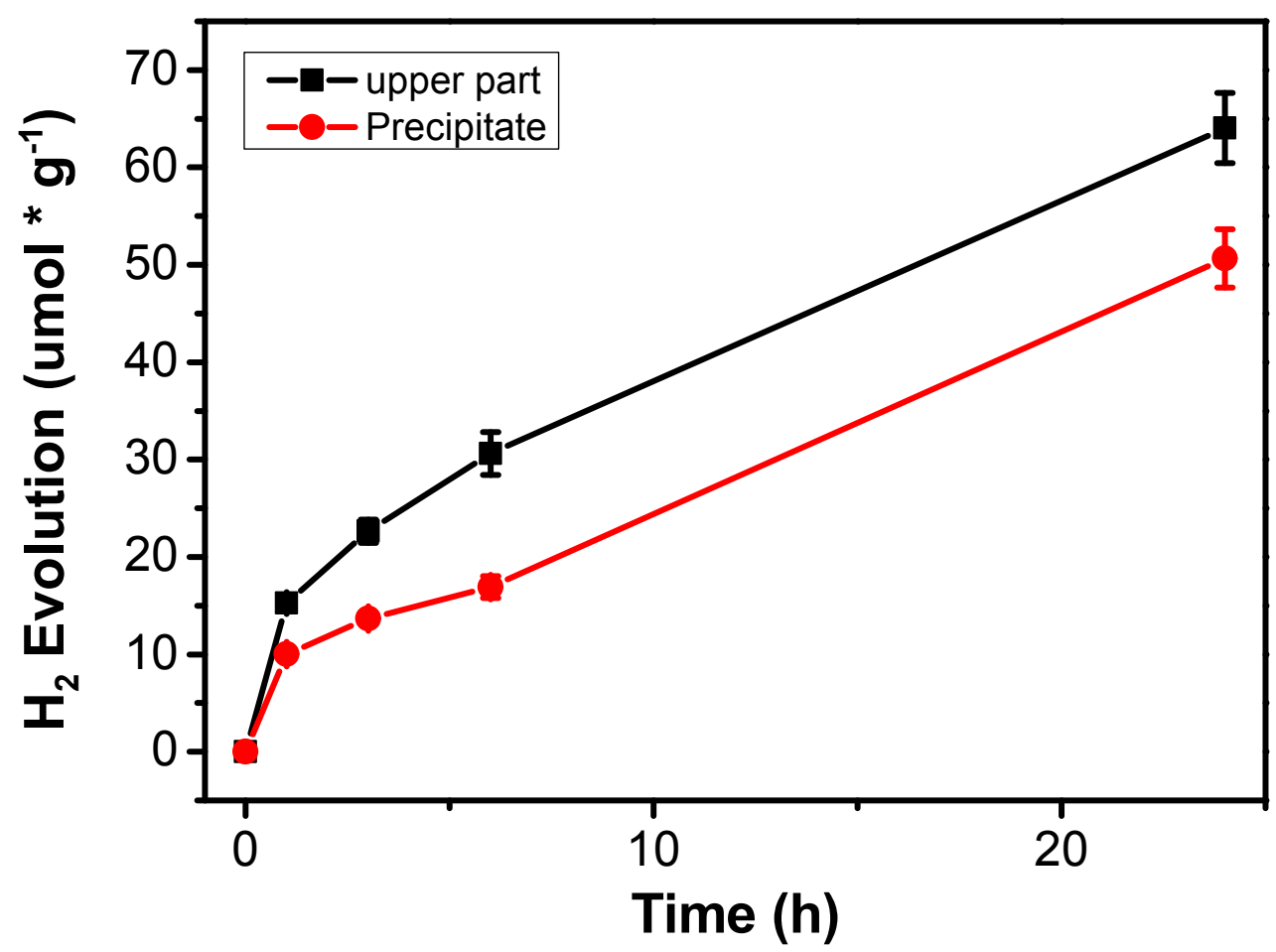

Figure S8. Photocatalytic activity of the upper and bottom material after dispersing 3D (N)C (10 $\mathrm{mg})$ in $\mathrm{H}_{2} \mathrm{O}(10 \mathrm{ml})$ ultrasounds and allowing to sediment the larger particles for $5 \mathrm{~h}$. Irradiation conditions: $300 \mathrm{~W}$ Xe lamp, 10 vol.\% TEAO as sacrificial electron donor, volume $10 \mathrm{~mL}$, ambient temperature.

Table S.1. List of different types of carbon and nitrogen family based on high resolution. experimental XPS peaks.

\begin{tabular}{|c|c|c|c|c|c|c|}
\hline Sample & $\begin{array}{c}\text { Graphitic C } \\
\text { [\%] }\end{array}$ & $\begin{array}{c}\mathrm{C}-\mathrm{O}, \mathrm{C}-\mathrm{N} \\
\mathrm{D}-[\%]\end{array}$ & $\begin{array}{l}\mathrm{N}-\mathrm{C}=\mathrm{O}, \\
\mathrm{O}=\mathrm{C}[\%]\end{array}$ & $\begin{array}{c}\text { Graphitic N } \\
{[\%]}\end{array}$ & $\begin{array}{c}\text { Pyridinic N } \\
{[\%]}\end{array}$ & $\begin{array}{c}\text { Oxisized } \\
\text { Pyrindinc } \\
\text { N [\%] }\end{array}$ \\
\hline $3 \mathrm{D}(\mathrm{N}) \mathrm{C}-1$ & 50.6 & 36.7 & 12.6 & 57.3 & 31.3 & 11.4 \\
\hline 3D (N)C-2 & 55.2 & 27.0 & 17.8 & 60.7 & 26.6 & 10.7 \\
\hline 3D (N)C-3 & 55.9 & 24.7 & 19.4 & 56.5 & 34.6 & 8.6 \\
\hline 3D (N)C-4 & 57.8 & 26.3 & 17.4 & 58.7 & 26.5 & 15.9 \\
\hline $3 \mathrm{D}(\mathrm{N}) \mathrm{C}$ & 56.2 & 34.4 & 9.6 & 61.5 & 26.3 & 10.3 \\
\hline
\end{tabular}


Table S.2. $\mathrm{CO}_{2}$ capture comparison of different porous carbon materials.

\begin{tabular}{|c|c|c|c|}
\hline Sample & $\begin{array}{c}\mathrm{S} \\
{\left[\mathrm{m}^{2} \mathrm{~g}^{-1}\right]}\end{array}$ & $\begin{array}{c}\mathrm{Q}_{\max } \\
{\left[\mathrm{mmol} \mathrm{g}^{-1}\right]}\end{array}$ & Ref. \\
\hline $3 \mathrm{D}(\mathrm{N}) \mathrm{C}-1$ & 477 & 2.96 & This work \\
\hline $3 \mathrm{D}(\mathrm{N}) \mathrm{C}-2$ & 499 & 3.03 & This work \\
\hline $3 \mathrm{D}(\mathrm{N}) \mathrm{C}-3$ & 402 & 2.61 & This work \\
\hline $3 D(N) C-4$ & 441 & 2.86 & This work \\
\hline GO & 4.27 & 0.166 & [1] \\
\hline GO-EDA-0.2 & 18.2 & 1.461 & [1] \\
\hline T-G-700-6 & 1296 & 2.52 & [2] \\
\hline GU-700-6 & 360 & 1.43 & [2] \\
\hline $\mathrm{P}-\mathrm{g}-\mathrm{C}_{3} \mathrm{~N}_{4}$ & 13.4 & 0.39 & [3] \\
\hline $\mathrm{g}-\mathrm{C}_{3} \mathrm{~N}_{4}$ & 3.0 & 0.12 & [3] \\
\hline CDMC & 871 & 1.31 & {$[4]$} \\
\hline
\end{tabular}

\section{Supplementary References}

1. An, L.; Liu, S.; Wang, L.; Wu, J.; Wu, Z.; Ma, C.; Yu, Q.; Hu, X., Novel Nitrogen-Doped Porous Carbons Derived from Graphene for Effective CO2 Capture, Ind. Eng. Chem. Res. 2019, 58, 3349-3358.

2. Cai, J.; Chen, J.; Zeng, P.; Pang, Z.; Kong, X., Molecular Mechanisms of CO2 Adsorption in Diamine-Cross-Linked Graphene Oxide, Chem. Mater. 2019, 31, 3729-3735.

3. Liu, B.; Ye, L.; Wang, R.; Yang, J.; Zhang, Y.; Guan, R.; Tian, L.; Chen, X., Phosphorus-Doped Graphitic Carbon Nitride Nanotubes with Amino-Rich Surface for Efficient CO2 Capture, Enhanced Photocatalytic Activity, and Product Selectivity, ACS Appl. Mater. Interf. 2018, 10, 4001-4009.

4. $\quad$ Peng, H.-L.; Zhang, J.-B.; Zhang, J.-Y.; Zhong, F.-Y.; Wu, P.-K.; Huang, K.; Fan, J.-P.; Liu, F., Chitosan-Derived Mesoporous Carbon with Ultrahigh Pore Volume for Amine Impregnation and Highly Efficient CO2 Capture, Chem. Eng. J. 2019, 359, 1159-1165. 